\title{
Can optical spectroscopy directly elucidate the ground state of $\mathrm{C}_{20}$ ?
}

\author{
Alberto Castro \\ Departamento de Física Teórica, Universidad de Valladolid, E-47011 Valladolid, Spain \\ Miguel A. L. Marques \\ Departamento de Física de Materiales, Facultad de Químicas, Universidad del País Vasco, Centro Mixto \\ CSIC-UPV/EHU and Donostia International Physics Center, 20080 San Sebastián, Spain \\ Julio A. Alonso \\ Departamento de Física Teórica, Universidad de Valladolid, E-47011 Valladolid, Spain \\ George F. Bertsch \\ Physics Department and Institute for Nuclear Theory, University of Washington, Seattle, Washington 98195 \\ K. Yabana \\ Institute of Physics, University of Tsukuba, Tsukuba 305-8571, Japan \\ Angel Rubio \\ Departamento de Física de Materiales, Facultad de Químicas, Universidad del País Vasco, CSIC-UPV/ \\ EHU and Donostia International Physics Center, 20080 San Sebastián, Spain
}

(Received 31 August 2001; accepted 6 November 2001)

The optical response of the lowest energy isomers of the $\mathrm{C}_{20}$ family is calculated using time-dependent density functional theory within a real-space, real-time scheme. Significant differences are found among the spectra of the different isomers, and thus we propose optical spectroscopy as a tool for experimental investigation of the structure of these important clusters.

(c) 2002 American Institute of Physics. [DOI: 10.1063/1.1430737]

Fullerenes are carbon clusters formed by the closing of a graphitic sheet; the needed curvature is supplied by the insertion, among a given number of graphitic hexagons, of 12 pentagons. ${ }^{1}$ Besides its most well-known representative, the nearly spherical $\mathrm{C}_{60}$, a wide variety of fullerenes has been predicted and experimentally observed. However, as we reduce the number of atoms, these structures become more reactive and unstable: The pentagons present in fullerenelike geometries, although being its major source of interest, are a cause of strain, especially if two of them are neighbors. Small fullerenes, with their high proportion of pentagons, have been actively sought and studied (see, e.g., Ref. 2). Recent discoveries include the synthesis and purification of the solid form of $\mathrm{C}_{36},{ }^{3}$ and the production of the cage and bowl isomers of $\mathrm{C}_{20} \cdot{ }^{4}$ The vibronic fine structure in photoelectron spectra of the cage has also been recently calculated, ${ }^{5}$ confirming the previous experimental assignation.

These medium-sized carbon clusters are predicted to possess a wide variety of isomers like cages, bowls, planar graphitic structures, rings, and linear chains. The theoretical and experimental study of the different isomers is important, because it may help us to better understanding the way fullerenes form. Several growth mechanisms have been proposed over the past years. ${ }^{6}$ In the so-called "pentagon road," fullerenes grow by the addition of small carbon fragments to bowl-like structures. The "fullerene road" is similar to the pentagon road, but the addition of the small carbon fragments is made to closed cagelike clusters. However, neither cages or bowls are usually seen in experiments. ${ }^{7}$ The most common technique for carbon cluster formation is laser va- porization of graphite and subsequent supersonic expansion into an inert gas atmosphere. At these high temperatures the preferred isomers are rings and chains. This led Jarrold and co-workers to propose a third path, namely that fullerenes grow by the coalescence and annealing of medium sized carbon rings. ${ }^{8}$

The smallest possible fullerene, consisting only of 12 pentagons with no graphitic hexagons intercalated, is the $\mathrm{C}_{20}$ cage isomer. ${ }^{9}$ Other low energy structures of $\mathrm{C}_{20}$ include a bowl (which may be considered a $\mathrm{C}_{60}$ fragment), several rings, and other closed 3D arrangements. Prinzbach et al. ${ }^{4}$ have recently reported the production of the cage and bowl members of the family. The smallest fullerene cannot be expected to form spontaneously, but has been produced from the similarly shaped precursor $\mathrm{C}_{20} \mathrm{H}_{20}$, after replacing hydrogen with $\mathrm{Br}$. The bowl was produced in the same way, and photoelectron spectroscopy was used to distinguish unambiguously between the different species.

It has not been possible to make reliable theoretical predictions of the most stable structure of $\mathrm{C}_{20}$. In fact, different levels of theories favor different geometries: at the HartreeFock level, the ring is the ground state, followed by the bowl and the cage. ${ }^{10}$ Density functional theory (DFT) ${ }^{11}$ in its local density approximation (LDA) reverses the order, giving the cage as the lowest energy structure. ${ }^{10,12}$ Another complication is that entropy effects can affect the relative stability. Molecular-dynamics simulations with the Car-Parinello method and the LDA show that increasing temperature changes the favored structure from cage to bowl, then to the 


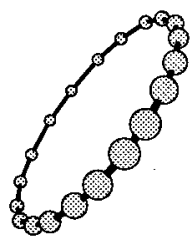

ring

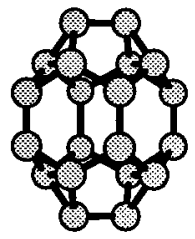

(d)

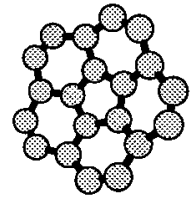

bowl



(e)
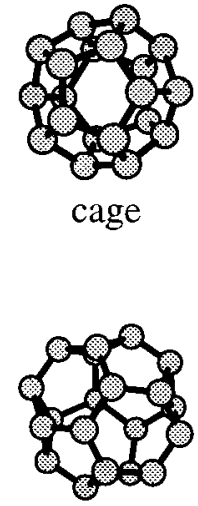

(f)
FIG. 1. Isomers of $\mathrm{C}_{20}$.

ring. ${ }^{13}$ Better functionals are available with the generalized gradient approximation (GGA), but their use does not clarify matters: the ordering of the isomers depends on the correction used. ${ }^{14,10,12}$ Quantum Monte Carlo (QMC) and coupled cluster (CC) methods have also been applied in an attempt to resolve the issue, yielding bowl-ring-cage ordering using the former method ${ }^{10}$ and cage-bowl-ring using the latter. ${ }^{15}$ Furthermore, it seems that the results are sensitive to the pseudopotential employed. ${ }^{15}$ Changing slightly the pseudopotential cutoff radius may actually reverse the ordering of the isomers.

Thus it is important to find an experimental method to determine the structure that is sensitive enough to be usable with the available cluster beam intensities. In this respect optical spectroscopy is a useful tool to characterize geometries. Some time ago, Rubio et al. ${ }^{16}$ proposed this technique to determine the structure of semiconductor and metal clusters. In particular, they showed that the optical absorption spectra of different isomers of $\mathrm{Si}_{4}$ and $\mathrm{Si}_{8}$ are sufficiently different to distinguish easily between them. The situation is similar with respect to carbon structures. The time-dependent density functional theory (TDDFT) was found to be quite reliable for determining the energies of the strong transitions in a variety of carbon structures ranging from chains ${ }^{17}$ to conjugated carbon molecules. ${ }^{18}$ In cases where the spectra could be compared with experiment, the lowest strong transition is typically reproduced to an accuracy of a few tenth of $\mathrm{eV}$. Comparing rings and chains, the transition energies differ by several $\mathrm{eV}$, easily allowing the structures to be distinguished. Thus it is quite promising to use the predicted excitation spectra of $\mathrm{C}_{20}$ structures to distinguish between them. In this spirit, the present study is aimed at the calculation of the optical response of six members of the $\mathrm{C}_{20}$ family (see Fig. 1): the smallest fullerene ("cage"), which is a Jahn-Teller distortion of a dodecahedron, the ring, the bowl, and three cagelike structures $(d),(e)$, and $(f)$. The structures $(d)$ and $(f)$, related by the Stone-Wales transformation, are quite regular, and composed of four hexagons, four pentagons, and four four-membered rings. These clusters are the six members with lower energy as calculated by
Jones ${ }^{19}$ within the LDA approximation. Other structures, such as bicycle rings and chains, may be favored by entropy at high temperature and have been observed experimentally. However, neither of them seem to be a possible ground state. We will show that calculations based on TDDFT predict characteristic optical spectra for the ring, cage, and bowl species.

We now describe briefly the methodology of the calculation of optical absorption in the TDDFT. We start with ground state structures and electron orbitals, determined with some implementation of DFT. This gives the initial condition for solving the time-dependent Kohn-Sham equation. Mathematically, there are several very different methods for solving the equation, but in principle the results should be the same if the energy functional is the same. Our method, solving the equation in real time and representing the wave function on a uniform spatial grid, is based on a nuclear physics algorithm, ${ }^{20}$ and has been described several times before. $^{12,18,21}$ The real-time response to an impulsive perturbation is Fourier transformed to get the dynamic polarizability in the entire range of interest. Of more direct physical interest is the optical absorption strength function $S(E)$, obtained from the imaginary part of the polarizability by the equation

$$
S(E)=\frac{2 m E}{\hbar^{2} e^{2} \pi} \operatorname{Im} \alpha(E) .
$$

With this definition, the $f$-sum rule is given by the integral $\int S(E) d N$.

For the Kohn-Sham energy functional, we used the LDA with the prescription of Ref. 22. Use of gradient corrections is possible within this framework, but results for the optical absorption have been reported to be quite insensitive to this change. ${ }^{23}$ Recent calculations of electronic excitations of a carbon-based molecule such as benzene ${ }^{24,25}$ also show a very close agreement. Slightly more important modifications are to be expected if exact-exchange functionals are used, although the qualitative differences among the spectra should remain. We also used a pseudopotential to avoid explicit consideration of the $1 s$ electrons, choosing the norm-conserving soft-core pseudopotential of Ref. 26. The numerical parameters that need to be specified for the calculation are mesh spacing, $0.25 \AA$; wave function domain, sphere of radius $8 \AA$ (slightly larger in the case of the ring); time step, $0.001 \hbar$ $\mathrm{eV}^{-1}$; number of time steps, 20000 . Thus the total propagation time is $T=20 \hbar \mathrm{eV}^{-1}$. One technical point that should be mentioned is that the Fourier transform over the finite interval $T$ gives peaks that are broadened by the time cutoff. In presenting the results, one removes the spurious oscillations associated with the time cutoff by multiplying by a filter function, amounting to a convolution in the frequency domain. The sum rule is preserved providing the filter function has zero slope at $t=0$. In any case, with $T=20 \hbar \mathrm{eV}^{-1}$, the individual states have a width of about $0.4 \mathrm{eV}$.

For the calculations reported here, we used structures from two sources. The geometries of the bowl, cage, and ring isomers were determined by Raghavachari et al. ${ }^{14}$ For the $(d),(e)$, and $(f)$ isomers, we used those obtained by Jones 
TABLE I. Ionization potential (IP), electron affinity (EA) and static dipole polarizability $(\alpha)$ of the $\mathrm{C}_{20}$ isomers. Experimental values of the electron affinity from Ref. 4 are given between parentheses.

\begin{tabular}{lccc}
\hline \hline & IP $(\mathrm{eV})$ & EA $(\mathrm{eV})$ & $\alpha\left(\AA^{3}\right)$ \\
\hline Ring & 7.8 & $2.6(2.44 \pm 0.03)$ & 51 \\
Bowl & 9.2 & $2.3(2.17 \pm 0.03)$ & 32 \\
Cage & 7.5 & $2.1(2.25 \pm 0.03)$ & 27 \\
$d$ & 8.4 & 1.8 & 28 \\
$e$ & 8.0 & 2.8 & 28 \\
$f$ & 7.9 & 3.0 & 28 \\
\hline \hline
\end{tabular}

from an all-electron density functional LSD calculation with an extended Gaussian basis set. ${ }^{19}$ As mentioned earlier, the energy differences between the isomers are quite sensitive to details of the energy functional and the pseudopotential. Fortunately that is not the case at all for the optical spectra. The optical response is quite insensitive to changes in the pseudopotential and in the energy functional, providing the structures do not change significantly. The optical spectra depend very much on the Kohn-Sham potential, but the differences in that are slight between the different parametrizations of DFT. The ionization potentials (IP) and electron affinities of the different structures are given in Table I. These quantities are calculated in the DFT by differences of total energies of systems with differing numbers of electrons but the same geometry. The results for the electronic affinity are good to within $0.2 \mathrm{eV}$ if compared to the experimental values reported by Prinzbach et al. in Ref. 4, which have been obtained through photoelectron spectra.

The results of our TDDFT calculations of the optical absorption are shown in Table II and Fig. 2. Table II gives the energies and strengths of the lowest transitions with appreciable strength, and Fig. 2 displays the strength function for energies up to the vacuum ultraviolet. The solid lines show the TDDFT results averaged over all orientations of the system. In the top panel we also show by a dashed line the single-electron response, which corresponds to difference of eigenvalues of the HOMO and LUMO orbitals. The dotted lines in the panels for the ring and bowl show the response perpendicular to the plane of the ring or the bowl center. This direction does not excite $\pi-\pi^{*}$ transitions in the ring and is almost negligible in the near ultraviolet frequencies (below 8 $\mathrm{eV})$, compared with the response within the plane. In the case of the bowl, the perpendicular response cannot be seen in the graph because of its extreme weakness in that energy range. This can be understood because there are no collective oscillations of the electrons in that direction. While present

TABLE II. Frequencies of selected peaks in the optical response of the studied structures, in eV. Between parentheses, estimations of their strengths.

\begin{tabular}{lcccccc}
\hline \hline & Ring & Bowl & Cage & $(d)$ & $(e)$ & $(f)$ \\
\hline$A$ & $5.20(5.4)$ & $5.05(0.7)$ & $3.88(0.2)$ & $2.47(0.03)$ & $3.77(0.1)$ & $3.53(0.1)$ \\
$B$ & $6.42(1.4)$ & $5.35(0.7)$ & $5.07(1.3)$ & $3.23(0.1)$ & $4.33(0.2)$ & $4.84(0.7)$ \\
$C$ & $7.09(2.0)$ & $6.60(0.7)$ & & $4.21(0.3)$ & $4.96(0.5)$ & $5.89(0.3)$ \\
$D$ & & $7.41(2.3)$ & & $4.67(0.4)$ & & \\
$E$ & & \multicolumn{5}{c}{$5.86(0.4)$} \\
\hline \hline
\end{tabular}

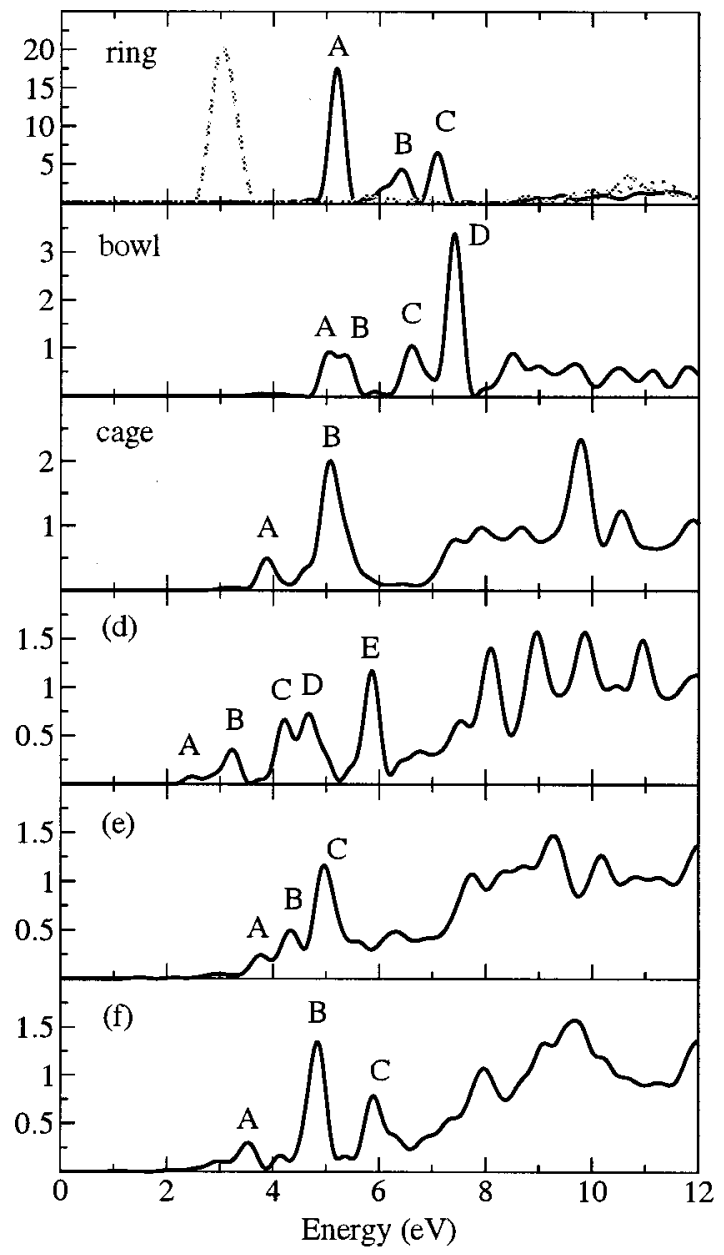

FIG. 2. Dipole strength function for the isomers of Fig. 1 , in $\mathrm{eV}^{-1}$, is shown by the solid line. The dashed line in the upper panel is the response in the independent particle approximation.

molecular beam experiments are not able to discriminate between the different spatial directions, the averaged spectra are still sufficiently different to discriminate between the different structures without ambiguity.

We can distinguish two regions in all the graphs: the peaks which can be seen in the near ultraviolet, and a broad absorption that starts at around $7.5 \mathrm{eV}$. The excitations responsible for this latter region are above the ionization threshold, which range from 7.5 to about $9 \mathrm{eV}$ for all cases considered, as can be seen from Table I. Since the LDA is unreliable for describing the ionization process (due to incorrect asymptotic potential), we will focus our attention on the relative positioning and intensity of the lower energy peaks.

The ring exhibits the largest gap in the spectrum and has the strongest collective transition. The bowl also has a high gap, larger than $5 \mathrm{eV}$, but the first significant transition is an order of magnitude weaker than in the ring. The relative intensities of the peaks, the fact that the first excitation is divided into two for the bowl, and the relative strength of the excitation in the $6-7 \mathrm{eV}$ region, can all be used to distinguish the bowl from the ring isomer.

The spectra of the four three-dimensional isomers start at much lower energy and are more similar to each other, which 
is expected from their similar geometries. The cage isomer shows two clear peaks at 3.9 and $5.1 \mathrm{eV}$, with the second much stronger than the first one. Most of the strength concentrates above the ionization threshold, and has a broad plateau starting at around $7 \mathrm{eV}$. This is clearly different from planarlike isomers, where an important fraction of the strength appears below $7 \mathrm{eV}$. Isomer $(d)$ can be distinguished by the presence of a transition at quite low energy, $2.5 \mathrm{eV}$, as well as by the fragmentation into many states going up to 6 $\mathrm{eV}$. Isomer $(e)$ differs from the cage by the presence of a transition $(B)$ between the transitions that would be seen in the cage. The spectrum of isomer $(f)$ is similar to the cage up to the second peak, but shifted down by about $0.3 \mathrm{eV}$. This is close to the borderline where the TDDFT energies are reliable. However, isomer $(f)$ also has a third peak near 6 $\mathrm{eV}$, in a region where there is a gap in the cage spectrum, and that difference would be definitive.

We also report calculations for the static polarizability in Table I. These have been calculated in two ways, by the formula

$$
\alpha(0)=\frac{e^{2} \hbar^{2}}{m} \int_{0}^{\infty} d E \frac{S(E)}{E^{2}},
$$

and by adding a static field to the DFT calculation. The two methods agree within $2 \%$, providing an additional check on our numerical TDDFT computations. We see that the predicted polarizabilities differ substantially between ring, bowl, and closed structure, but there is little discrimination among the closed structures.

In conclusion, we have found that the optical absorption spectra calculated in the TDDFT for different candidate structures of $\mathrm{C}_{20}$ show marked differences that could be used for structural determination. Some differences appear already in the visible and near ultraviolet, and complete discrimination should be possible with a measurement of the spectrum extending up to the $6 \mathrm{eV}$ region.

\section{ACKNOWLEDGMENTS}

This work was partially supported by the RTN program of the European Union NANOPHASE (Contract No. HPRN-
CT-2000-00167), DGESIC (PB98-0345), and JCyL (VA28/ 99). A.C. acknowledges support from the MEC under the graduate fellowship program, and hospitality from the INT at the University of Washington, G.B. acknowledges support by the U.S. Department of Energy under Contract No. E-FG-0690ER-41132. Most of the calculations were done at the Centro Europeo de Paralelismo de Barcelona (CEPBA).

${ }^{1}$ H. W. Kroto, Int. J. Mass Spectrom. Ion Processes 138, 1 (1994).

${ }^{2}$ A. van Orden and R. J. Saykally, Chem. Rev. 98, 2313 (1998).

${ }^{3}$ C. Piskotti, J. Jarger, and A. Zettl, Nature (London) 393, 771 (1998).

${ }^{4}$ H. Prinzbach, A. Weiler, P. Landenberger, F. Wahl, J. Wörth, L. T. Scott, M. Gelmont, D. Olevano, and B. v. Issendorff, Nature (London) 407, 60 (2000).

${ }^{5}$ M. Saito and Y. Miyamoto, Phys. Rev. Lett. 87, 035503 (2001).

${ }^{6}$ N. S. Goroff, Acc. Chem. Res. 29, 77 (1996).

${ }^{7}$ G. Von Helden, M. T. Hsu, N. G. Gotts, P. R. Kemper, and M. T. Bowers, Chem. Phys. Lett. 204, 15 (1993); A. K. Ott, G. A. Rechsteiner, C. Felix, O. Hampe, M. F. Jarrold, R. P. Van Duyne, and K. Raghavachari, J. Chem. Phys. 109, 9652 (1998).

${ }^{8}$ J. M. Hunter, J. L. Fye, E. J. Roskamp, and M. F. Jarrold, J. Phys. Chem. 98, 1810 (1994).

${ }^{9}$ M. F. Jarrold, Nature (London) 407, 26 (2000).

${ }^{10}$ J. C. Grossmann, L. Mitas, and K. Raghavachari, Phys. Rev. Lett. 75, 3870 (1995).

${ }^{11}$ W. Kohn and L. Sham, Phys. Rev. 140, A1133 (1965).

${ }^{12}$ R. O. Jones and G. Seifert, Phys. Rev. Lett. 79, 443 (1997).

${ }^{13}$ C. J. Brabec, E. B. Anderson, B. N. Davidson, S. A. Kajihara, Q. M. Zhang, J. Bernholc, and D. Tománek, Phys. Rev. B 46, 7326 (1992).

${ }^{14}$ K. Raghavachari, D. L. Strout, G. K. Odom, G. E. Scuseria, J. A. Pople, B. G. Johnson, and P. M. W. Gill, Chem. Phys. Lett. 214, 357 (1993).

${ }^{15}$ E. J. Bylaska, P. R. Taylor, R. Kawai, and J. H. Weare, J. Phys. Chem. 100, 6966 (1996).

${ }^{16}$ A. Rubio, J. A. Alonso, X. Blase, L. C. Balbás, and S. G. Louie, Phys. Rev. Lett. 77, 247 (1996).

${ }^{17}$ K. Yabana and G. F. Bertsch, Z. Phys. D: At., Mol. Clusters 42, 219 (1997).

${ }^{18}$ K. Yabana and G. F. Bertsch, Int. J. Quantum Chem. 75, 55 (1999).

${ }^{19}$ R. O. Jones, J. Chem. Phys. 110, 5189 (1999).

${ }^{20}$ H. Flocard, S. E. Koonin, and M. S. Weiss, Phys. Rev. C 17, 1682 (1978).

${ }^{21}$ K. Yabana and G. F. Bertsch, Phys. Rev. B 54, 4484 (1996); Phys. Rev. A 60, 1271 (1999); 60, 3809 (1999).

${ }^{22}$ J. P. Perdew and A. Zunger, Phys. Rev. B 23, 5048 (1981).

${ }^{23}$ M. A. L. Marques, A. Castro, and A. Rubio, J. Chem. Phys. 115, 3006 (2001).

${ }^{24}$ G. F. Bertsch, A. Schnell, and K. Yabana, J. Chem. Phys. 115, 4051 (2001).

${ }^{25}$ H. Heinze, A. Goerling, and N. Roesch, J. Chem. Phys. 113, 2088 (2000).

${ }^{26}$ N. Troullier and J. L. Martins, Phys. Rev. B 43, 1993 (1991). 